\title{
COMPOSIÇÃO E PROPRIEDADES FISIOLÓGICAS E FUNCIONAIS DA AVEIA
}

\section{COMPOSITION AND FUNCTIONAL PHYSIOLOGICAL PROPERTIES OF THE OAT}

\author{
Bruno Eduardo Malanchen ${ }^{1}$, Flavio Aparecido Da Silva ${ }^{2}$, Thainara Gottardi ${ }^{3}$, Daiana \\ Alves Terra ${ }^{2}$, Daniela Miotto Bernardi ${ }^{*}$
}

\footnotetext{
${ }^{1}$ Profissional de Educação Física. Especialista em Medicina Desportiva e acadêmico do curso de Nutrição do Centro Universitário FAG. ${ }^{2}$ Profissional de Educação e acadêmico do curso de Nutrição do Centro Universitário FAG. ${ }^{3}$ Acadêmicas de Nutrição do Centro Universitário FAG

${ }^{4}$ Nutricionista. Doutora em Alimentos de Nutrição. Professora Docente Nutrição o Centro Universitário FAG

*Autor correspondente: danimiottober@gmail.com, https://orcid.org/0000-0001-9019-3835
}

\section{RESUMO}

O consumo da aveia tem apresentado benefícios à saúde humana em diversas situações, seja pela redução do risco de complicações de algumas doenças ou pela melhora no quadro das mesmas nos indivíduos. Este cereal é altamente produzido no Brasil e no mundo e sua utilidade se dá de diversas maneiras, podendo ser útil para o consumo humano ou animal e até mesmo para a fabricação de cosméticos. A aveia possui uma composição nutricional variada e repleta de nutrientes que apresentam benefícios à saúde humana, sendo eles compostos antioxidantes, fenólicos, bglucana, boa quantidade de aminoácidos, fibras, e carboidratos. Essa composição nutricional tem apresentado variações de acordo com sua produção, processamento, tratamento térmico e consumo, sendo necessários mais estudos para avaliar estes efeitos na saúde humana. O objetivo deste trabalho foi realizar uma revisão bibliográfica de literatura, de característica retrospectia e descritiva, utilizando-se de dados à partir das bases de dados PUBMED, MEDLINE, Scielo e Google Acadêmico, sobre a produção deste cereal e seus diversos efeitos sobre a saúde humana através de seu consumo. Palavras-Chave: Aveia; Composição Nutricional; Compostos Bioativos; B-Glucana; Doenças.

\begin{abstract}
The consumption of oats has shown benefits to human health in several situations, either by reducing the risk of complications of some diseases or by improving them in individuals. This cereal is highly produced in Brazil and in the world and its usefulness comes in many different ways, it can be useful for human or animal consumption and even for the manufacture of cosmetics. Oats have a varied nutritional composition and are full of nutrients that have benefits to human health. They are composed of antioxidants, phenolics, b-glucans, a good amount of amino acids, fibers and carbohydrates. This nutritional composition has presented variations according to its production, processing, thermal treatment and consumption, being necessary more studies to evaluate these effects in human health. The objective of this work was to carry out a bibliographical review of literature, with retrospective and descriptive characteristics, using data from PUBMED, MEDLINE, Scielo and Google Academic databases on the production of this cereal and its various effects on health through their consumption. Keyboards: Oatmeal; Nutrition Composition; Bioactive Compounds; B-Glucan; Diseases.
\end{abstract}




\section{INTRODUÇÃO}

Na produção agrícola brasileira da atualidade, a demanda por cultivares de alta produtividade é cada vez maior, para que com isso obtenham-se produtos de boa qualidade e potencial comercialização. A aveia-branca (Avena sativa L.) vem apresentando uma grande importância como cultura alternativa de inverno com uma ampla área de cultivo no Brasil, principalmente na região Sul do País (CRESTANI et al., 2010).

A aveia é uma gramínea anual pertencente a família Poaceae, tribo Aveneae e gênero Avena. Tal gênero compreende várias espécies silvestres, daninhas e cultivadas distribuídas em seis continentes (GUTKOSKI e PEDÓ, 2000). As principais espécies cultivadas no país são a aveia branca (Avena sativa L.), esta que é prioritamente utilizda no consumo humano, a aveia amarela (Avena byzantina C. Koch), espécies de duplo propósito com produção de forragem e grãos, e a aveia preta (Avena strigosa Schreb) empregada como pastagem, de forma isolada ou em consorciação com outras forrageiras, e como adubo verde.

O cereal possui múltiplas formas de utilização: produção de grãos para consumo humano; matéria prima industrial para a produção de cosmético e insumos para indústria química; consumo animal de grãos ou para formação de pastagens de inverno para pastejo e/ou elaboração de feno e de silagem, cobertura de solo, adubação verde com vistas a implantação das culturas de verão, em sucessão. O uso na alimentação animal constitui seu maior uso no Brasil e no mundo. Quando empregada em forma de ração, a maior demanda é para uso na alimentação de cavalos de corrida. $\mathrm{Na}$ alimentação humana, o cereal tem sido empregado na produção de alimentos infantis, cereais matinais (quentes ou frios), granola, muslins, barra de cereais, produtos forneados ou assados (pães, biscoitos, bolos, etc.), componente adicional para engrossar sopas, molhos e para aumentar o volume de produtos cárneos (DE MORI, 2012).

Diversas doenças têm apresentado melhora ou redução do risco por meio do consumo da aveia ou de produtos que contenham ela em sua composição. Dentre as doenças destacam-se principalmente o diabetes mellitus e suas complicações sobre a glicemia, onde o consumo de aveia pode diminuir a absorção de glicose, o que é benéfico para diabéticos, e pode estimular funções imunológicas, tanto in vitro quanto in vivo (DE MORI, 2012). O uso da farinha e do farelo de aveia tem crescido e auxiliado no controle de doenças, conforme supracitado, principalmente pela presença de um elevado teor de polissacarídeos, proteínas, minerais e lipídeos benéficos à saúde (MOLIN, 2011).

Entre os polissacarídeos presentes neste cereal, destacam-se as beta-glucanas (B-Glucana), componentes estruturais das paredes celulares dos cereais, que atuam na redução do colesterol em indivíduos com hipercolesterolemia, e consequentemente na diminuição dos riscos de doenças cardiovasculares e suas respectivas complicações (DE MORI, 2012).

A B-Glucana consumida por indivíduos com sobrepeso e obesidade (compostos por inulina, beta-glucano de aveia e antocianinas e polifenóis de mirtilo), onde melhorou a tolerância à glicose e reduziu glicemia pós-prandial, além de causar alterações nos marcadores fecais, diminuir o pH das fezes e aumentar a sensação de saciedade e a flatulência. A diminuição do $\mathrm{pH}$ e o aumento da flatulência são indicadores de fermentação. Além disso, houve um aumento nas concentrações plasmáticas do peptídeo YY (PYY) e uma diminuição nas concentrações plasmáticas 
de grelina, o que surte efeito positivo e mantém a saciedade por mais tempo (REBELLO, 2015).

Como citado anteriormente, o consumo deste cereal pode ser realizado de diferentes formas, com isso e o mesmo poderá proporcionar efeitos diferentes ao organismo humano e suas mais variadas situações fisiológicas apresentadas, pois os nutrientes que podem apresentar benefícios em determinadas funções do corpo humano poderão se apresentar em diferentes concentrações dependendo de como o alimento será preparado e consumido (MOLIN, 2011).

Sendo assim, o objetivo deste trabalho é avaliar por meio da literatura a eficácia do uso da aveia na dieta e a influência de seus compostos químicos e nutricionais sobre a saúde humana em determinadas funções fisiológicas.

\section{METODOLOGIA}

Trata-se de uma pesquisa bibliográfica, retrospectiva, de natureza descritiva, com abordagem quantitava e ênfase na produção científica acerca das temáticas em questão (GIL, 2002).

Foram utilizadas fontes de dados a partir das bases de dados PUBMED, MEDLINE, Scielo e Google Acadêmico, sendo utilizados como indexadores de bubsca as palavras-chave "oatmeal", "nutrition composition", "bioactive compounds", " $B$ Glucan" e "disease".

As palavras chaves foram utilizadas separadamente nas bases de dados afim de que os artigos buscados tivessem maior relação com os temas propostos para esta pesquisa de revisão.

$\mathrm{Na}$ base de dados foram buscados os indexadores "oatmeal", assim como juntamente com as demais palavras-chave. Na base de dados PUBMED para "oatmeal + nutrition composition" foram encontrados 9 artigos, para "oatmeal + bioactive compounds" foram encontrados 2 artigos, já para "oatmeal + b-glucan" foram encontrados 11 artigos e para "oatmeal + disease" foram encontrados 89 artigos.

Na base de dados MEDLINE: para "oatmeal" foram encontrados 105 artigos, para "oatmeal + disease" 28 artigos, para "oatmeal + b-glucan", 22 artigos, para "oatmeal + bioactive compounds" e "oatmeal + nutrition composition" não foram encontrados artigos na busca.

$\mathrm{Na}$ base de dados Scielo foram utilizados os indexadores de busca na língua portuguesa, sendo que para a palavra "aveia" foram encontrados 1065 artigos, "aveia + composição nutricional" 14 artigos, para "aveia + b-glucana" 1 artigo, "aveia + doenças" 26 artigos". Para "aveia + compostos bioativos" não foram encontrados artigos.

Para a base de dados do Google Acadêmico foram utilizados os indexadores novamente na língua inglesa, sendo que para "oatmeal + disease" foram encontrados 19700 artigos, "oatmeal + b-glucan" 2700 artigos, "oatmeal + nutrition composition" 13700 artigos, "oatmeal + bioactives compounds" 17700 artigos.

Foram encontrados, ao todo, 55172 artigos, sendo utilizados 44 artigos para elaboração desta pesquisa. Como critérios de inclusão foram incluídos os artigos na língua inglesa e portuguesa, publicados em um período de até 33 anos (entre 1986 e 2019), que contemplassem ou que fornecessem informações compatíveis com o objetivo do trabalho. Após a identificação dos artigos considerados relevantes para o tema proposto, foi realizada a análise descritiva dos mesmos e ponderações foram efetuadas, com respeito à confiabilidade e generalizabilidade dos métodos diagnósticos utilizados. 


\section{COMPOSIÇÃO BROMATOLÓGICA E COMPOSTOS BIOATIVOS}

$\mathrm{Na}$ produção agrícola brasileira atual é cada vez maior a necessidade por cultivares com alto rendimento de produção, que ainda possibilitem a produção de produtos com qualidade elevada e boa aceitação comercial. A aveia-branca (Avena sativa L.) tem assumido grande importância como cultura de cultivo de inverno no Brasil, principalmente na região sul do país. A composição química e estrutural do grão da aveia é única entre os cereais, o que lhe confere aptidão para uso na alimentação humana. É um alimento com excelente valor nutricional, rico em fibras alimentares solúveis, sendo uma das mais importantes a B-glucana, que é responsável por relevantes benefícios à saúde humana, conferindo para este cereal a condição de alimento funcional (CRESTANI et al., 2010). Além disso também se destacam a qualidade proteica, variando entre 12,40 a $24,50 \%$ no grão descascado, e a sua maior porcentagem de lipídios, que varia de 3,10 a 10,90\%, distribuídos por todo o grão e com predominância de ácidos graxos insaturados, sendo os dois principais o oléico e o linoléico, e ácidos graxos saturados, sendo o principal encontrado o ácido palmítico, e ainda, o montante somado por estes três ácidos graxos tendem a representar em torno de 95\% do total (WEBER, et al, 2002).

Zhou et al (1998), estudou o efeito do ambiente em oito cultivares diferentes de aveia provenientes do Norte, Centro e Sul da Austrália. Foram quantificados 13 ácidos graxos, aparecendo em menor proporção os ácidos mirístico, esteárico e linolênico. As maiores concentrações dos ácidos graxos oléico e linoléico foram de 37,9\%-42,6\% e 35,9\%-39,9\%, respectivamente. O palmítico foi o ácido graxo saturado em maior quantidade, variando entre 17,0\%-19,3\%. Os autores concluíram que o ambiente exerce influência sobre o conteúdo dos ácidos graxos e o fator varietal contribui com mais de $70 \%$ da variação dos ácidos graxos nas diferentes cultivares sobre a composição dos ácidos graxos.

Segundo Crestani et al (2010), a composição de fibras alimentares da aveia varia suas quantidades de acordo com diferentes locais de cultivo, sendo assim, sugere-se que o solo, o clima, o modo de cultivo e as interferências climáticas podem interferir na qualidade nutricional do alimento, podendo não somente mudar as quantidades de fibras, mas também de outros nutrientes.

Além de possuir um alto teor de lipídeos benéficos à saúde e também um teor proteico elevado quando comparada com o trigo e outros cereias, a aveia possui uma boa composição de aminoácidos, sendo encontrados na Avena Sativa L, os seguintes aminoácidos: valina, isoleucina, leucina, treonina, 1/2 cristina, metionina, tirosina, fenilalanina, histidina, lisina, triptofano, ácido aspártico, serina, ácido glutâmico, prolina, glicina, alanina. Os aminoácidos Lisina e Treonina acabam sendo os principais aminoácidos limitantes na composição da aveia (WEBER, et al, 2002).

Com relação à digestibilidade da proteína, fator que também avalia seu valor nutritivo, a cultivar apresentou entre $86,12 \%$ e $98,86 \%$ de digestibilidade (GUTKOSKI, EL-DASH, PEDÓ, 1997; WEBER, et al, 2002).

Este cereal é rico em uma grande variedade de compostos fenólicos com atividade antioxidante comprovada in vitro e in vivo e, além disso, também se enquadra na definição de "grão integral" por apresentar, após o seu processamento, o mesmo balanço de nutrientes encontrado na matéria-prima original (KLAJN, 2012).

Dentre os compostos antioxidantes destacam-se, especialmente compostos fenólicos, principalmente os flavonóides. A concentração de fenólicos pode ser afetada por vários fatores como genótipo, tratamento térmico e tipo de 
processamento. Os ácidos ferúlico e caféico e seus ésteres foram os compostos inicialmente identificados e estudados. Vitamina $E$ (tocoferol), ácido fítico, e a avenantramida presente exclusivamente na aveia com as variações dos tipos 1, 3, e 4, são os compostos antioxidantes mais abundantes e biodisponíveis na aveia, além de terem propriedades anti-inflamatórias e anti-aterogênicas. (DYKES, L. ROONEY, LW, 2007; KLAJN, 2012). Os compostos fenólicos da aveia contêm uma mistura de derivados dos ácidos benzóico e cinâmico, quinonas, flavonas, flavonóis, flavononas, antocianinas e aminofenólicos. Os principais ácidos livres são o ácido caféico, ácido siríngico, ácido ferúlico e ácido sinápico (KLAJN, 2012).

A aveia se destaca como uma das principais fontes de compostos que exibem atividade antioxidante em grãos, porém a preservação dos compostos fenólicos é dependente de condições de processamento, por isso é importante à indústria monitorar as variáveis empregadas no tratamento hidrotérmico para que se obtenham produtos, que além de nutritivos, sejam ricos em compostos fenólicos com atividade antioxidante, benéficos à saúde, porém, ainda há um certo grau de carência de estudos que tragam como as condições de processamento e as características tecnológicas interferem sobre a atividade antioxidante deste grão (KLAJN, 2012).

\section{PROPRIEDADES FUNCIONAIS E FISIOLÓGICAS}

\subsection{AVEIA E DIABETES}

Diabetes é uma doença crônica caracterizada pela produção insuficiente de insulina ou pelo aumento da resistência a mesma, com impactos negativos sobre o metabolismo de carboidratos em primeira instância podendo causar consequências negativas para a saúde do indivíduo, quando não controlada. O controle da glicemia é considerado o principal objetivo do diabetes, buscando o consumo adequado, moderado e fracionado de carboidratos (SILVA; MELO, 2006).

O diabetes tipo 2 é uma doença crônica comum, com grande carga global de saúde e economia. A prevalência ainda é crescente devido a mudanças no estilo de vida, especialmente em países em desenvolvimento (SHAW, et al, 2010; ZHANG, et al, 2010).

Todavia, as diretrizes da Associação Americana de Diabetes - ADA (2002), não fazem restrições ao consumo de qualquer tipo de carboidratos. Baseados em critérios adotados de dose diária recomendada (do inglês: Recommendad Dietary Allowances - RDA), publicada pelo Departamento de Agricultura dos Estados Unidos (do inglês: US Departament of Agriculture- USDA), a ADA recomenda que a ingestão mínima de carboidratos deve ser de 130g/dia.

Os grãos de cereais inteiros intactos têm-se revelado eficaz na redução de glicose e respostas de insulina. Outro fator interessante, é que a expressão de GLP1 também mostrou aumento de saciedade, o que seria muito interessante para pacientes diabéticos levando em conta o controle do apetite e consequentemente a manutenção da sensibilidade à insulina (BRITISH JOURNAL OF NUTRITION, 2013).

A educação dietética, a terapia nutricional, a atividade física, a farmacoterapia e o monitoramento da glicose são componentes fundamentais do controle do diabetes. A intervenção no estilo de vida, incluindo o controle da dieta, é recomendada como a abordagem fundamental para todos os pacientes com diabetes tipo 2 . Sugere-se que os pacientes diabéticos consumam pelo menos a quantidade de fibras e grãos integrais recomendados para o público em geral, que é de $14 \mathrm{~g}$ de fibra / $1000 \mathrm{kcal}$ por dia ou $25 \mathrm{~g} /$ dia para mulheres adultas e $38 \mathrm{~g} /$ dia para homens adultos (EVERT, 
2014). Para a doença ser tratada, tembém necessário o uso de medicamentos, como hipoglicemiantes orais ou insulina, e também reeducação alimentar (SOCIEDADE BRASILEIRA DE DIABETES, 2003).

Alimentos com fibras dietéticas solúveis tem numerosos benefícios para a saúde de seres humanos, incluindo a redução dos níveis de glicose no sangue pósprandial, e melhorando a resposta global da glicemia ( LU ZX, 2004), e sensibilidade à insulina (BODINHAM, 2013), por isso são ótimas alternativas para indivíduos com DM. São ações benéficas para a digestão e absorção de macronutrientes no intestino delgado e resultam em menores respostas da glicemia e insulina pós-prandial (CHRISTENSEN, 2013).

A aveia é uma boa fonte de fibra dietética solúvel rica em B-glucana, que é considerada um componente bioativo na redução de respostas pós-prandiais de glicose e insulina, atuando na melhora da sensibilidade à insulina, mantendo 0 controle glicêmico e regulando os lipídios sanguíneos (BRENNAN, 2005; WANG 2014). A FDA (Do inglês: Food and Drug Administration dos Estados Unidos ) sugeriu que o consumo de $3 \mathrm{~g}$ ou mais por dia de $\mathrm{B}$-glucana de aveia ou cevada pode reduzir o risco de doença cardíaca coronária (FDA, 2015).

A quantidade de fibras na alimentação é um dos pilares de uma alimentação saudável, onde se recomenda um consumo diário de no mínimo 25g/dia de fibras (BRASIL, 2005).

Jacintho (2007) mostrou em seu trabalho a recomendação internacional da Associação Dietética Americana (ADA), onde o consumo de fibras alimentares indicado para adultos deve ser de 20 a $35 \mathrm{~g} / \mathrm{dia}$. Para idades entre 2 a 20 anos, a recomendação é de que se acrescente $5 \mathrm{~g}$ de fibras à idade. No Brasil a recomendação da Agência de Vigilância Sanitária (ANVISA, 2008) é de que os alimentos contenham 3 gramas de B-glucana na porção do alimento sólido pronto para o consumo, ou de 1,5 gramas no alimento líquido (em torno de $150 \mathrm{kcal}$ ).

De modo geral essas fibras estão relacionadas à prevenção de diabetes, doenças cardiovasculares, obesidade, auxilio na perda de peso, aumento da saciedade, promoção da redução de colesterol e triacilglicerol plasmático, diminuição a glicemia pós-prandial, entre outras funções (PHILIPPI, 2008).

O consumo de fibras solúveis e insolúveis está associado à melhora da resistência à insulina, sendo que o maior consumo de fibras aumenta a sensibilidade à insulina em indivíduos saudáveis ou portadores de diabetes mellitus (MILANE, 2012).

Os efeitos fisiológicos das fibras alimentares não dependem somente da natureza química, mas também da estrutura física de seus constituintes, como peso molecular, tamanho de partícula e grau de esterificação. A administração aguda de fibras pode causar efeitos imediatos, assim como a administração crônica pode surtir efeito dentro de algum tempo. Os mecanismos através dos quais as fibras alimentares afetam o metabolismo glicídico, estão associados a alterações no trânsito, na morfologia e nos processos digestivos e absortivos gastrointestinais (REYES \& AREAS, 2001).

De acordo com relatório recente da Organização Mundial da Saúde (OMS) sobre dieta, nutrição e prevenção de doenças crônicas não transmissíveis (DCNT), o consumo alimentar habitual constitui um dos principais fatores determinantes passíveis de modificação para DCNT. Embora as evidências epidemiológicas demonstrem um potencial efeito protetor do elevado consumo de fibras e teores reduzidos de índice glicêmico da dieta habitual para o diabetes, a influência da 
qualidade dos carboidratos na etiologia dos distúrbios do metabolismo da glicose ainda é pouco compreendida.

A B-glucana da aveia é conhecido pelo seu efeito na redução dos níveis pósprandiais de glicose e insulina após carga oral glicêmica em pacientes diabéticos. Tanto a goma isolada da aveia, assim como o farelo da aveia, contém o B-glucana têm se mostrado benéfico. Fatores Intrínsecos de um alimento podem influenciar, o seu impacto na glicemia, dentre os quais a sua forma física, o grau de processamento, o tipo de amido, e a preparação, bem como o tipo específico ou variedade do alimento SILVA (2006).

Além da presença de B-glucana a aveia também tem seu papel perante o Diabetes Melitus pelo fato de possuir um baixo índice glicêmico, o que auxilia na doença reduzindo os picos glicêmicos pós-prandiais precoces e também diminuindo o risco de hipoglicemia pós-absortiva em pacientes com esse tipo de patologia (LUDWIG, 2002).

Segundo Silva, el al, (2006), em uma revisão de literatura, mostrou que o manejo dietoterápico do diabetes deve ter como principais objetivos: a redução da hiperglicemia, a prevenção da hipoglicemia e a diminuição do risco de complicações, principalmente também as cardiovasculares., concluiu que o consumo de B-glucana e de dietas ricas em fibras é essencial para o tratamento e a prevenção do diabetes devido ao papel perante a redução da velocidade de absorção da glicose e e também pelo decréscimo da produção de insulina no pâncreas, diminuindo assim os picos na glicemia dos pacientes. Há ainda divergência na literatura sobre a quantidade a ser consumida, porem sugere-se a ingestão de 2,5 a $3 \mathrm{~g}$ ao dia.

Pereira et al (2011) avaliaram o consumo de biscoitos com a adição de soja e aveia em ratos diabéticos. Foram elaboradas três formulações de biscoitos, contendo farinha de soja e aveia e foi realizada a análise sensorial das formulações com indivíduos diabéticos não treinados, utilizando o método afetivo com escala hedônica. Também foi realizado um ensaio biológico que utilizou-se de 24 ratos Wistar, divididos em grupos de seis animais: Grupos I e II, testes, compostos por animais alimentados com dieta adicionada de $20 \%$ do produto elaborado; Grupos III e IV, controles, compostos por animais alimentados com dieta padrão. Os animais dos grupos I e III foram induzidos ao diabetes. As análises constataram menores níveis glicêmicos, menor ganho de peso, além de controle no volume de excreção fecal, o que possivelmente seria pelo teor de fibras presente na aveia. De forma geral, o consumo dos biscoitos apresentaram efeitos benéficos sobre o diabetes.

Brandão et al (2005) mostraram através da Diretriz Brasileira de Diagnóstico e Tratamento da Síndrome Metabólica e suas complicações (maior resistência à insulina levando ao diabetes é uma delas) traz a informação de que a adoção de um plano alimentar saudável é fundamental no tratamento e na prevenção, recomendando então a ingestão diária de $20 \mathrm{~g}$ a $30 \mathrm{~g}$ de fibras (selecionando preferencialmente alimentos integrais ou minimamente processados com baixo índice glicêmico).

\subsection{AVEIA E TRATO GASTRO INSTESTINAL}

Para Reis (2003), as fibras alimentares são polissacarídeos (celulose, hemicelulose, pectinas, gomas, mucilagens) e polímeros de fenóis (lignina) componentes dos alimentos (cereais, grãos, raízes e folhas), que são resistentes à ação das enzimas digestivas no trato gastro intestinal humano. Essas fibras podem ser parcialmente digeridas por bactérias do cólon ou excretadas de forma inalterada 
nas fezes. A fibra alimentar compreende uma ampla variedade de substâncias com diferentes propriedades químicas, físicas e fisiológicas. Estas substâncias são provenientes principalmente da parede celular de plantas. Consideradas como alimentos funcionais, ajudam a melhorar as atividades vitais e o bom desempenho do trato gastrointestinal.

Distúrbios e doenças gastrointestinais como a síndrome do cólon irritável, hérnia de hiato, apendicite, diverticulite, doença de Crohn, hemorróidas e constipação intestinal têm sido correlacionadas com a ingestão de fibra alimentar, que segundo a Organização Mundial de Saúde e a FDA deve ser de 27 a 40g/dia e 25 a 35 g/dia, respectivamente para homens e mulheres. Embora a etiologia desses distúrbios e doenças não tenha sido atribuída unicamente à ingestão reduzida de fibra na alimentação, o aumento da ingestão de fibra alimentar tem sido considerado de fundamental importância para reduzir as complicações causadas por essas doenças (PACHECO \& SGARBIERI, 2001). Para Reis (2003) as recomendações diárias são de 20 a 50 gramas.

A fibra é de grande relevância para a comunidade em geral, sendo facilmente encontrada em grãos como soja, aveia e em cascas dos alimentos em geral. Segundo a Tabela Brasileira de Composição de Alimentos (TACO, 2011), a aveia possui uma quantidade de fibras de $9,1 \mathrm{~g}$ em $100 \mathrm{~g}$ de alimento.

A constipação intestinal acomete cerca de $20 \%$ da população mundial e constitui um dos sintomas mais frequentes de procura ao médico. É mais comum em mulheres e idosos e se encontra entre as doenças funcionais do intestino. Pode ser referida pelo paciente como fezes endurecidas, esforço excessivo no ato evacuatório, evacuações infrequentes ou sensação de evacuação incompleta. Subdivide-se em primária e secundária, tendo essa última causa bem definida, como doenças endócrinas e neurológicas ou uso inadvertido de substâncias obstipantes. É importante que as pessoas saibam da necessidade de uma dieta rica em fibras e de uma hidratação adequada (ALVES, 2012).

Chaud et al (2017) avaliou o consumo de fibras solúveis e insolúveis na dieta de camundongos e observou menor ganho de peso no crescimento dos camundongos alimentados com uma dieta padrão enriquecida com fibras insolúveis. Já para camundongos alimentados com uma dieta padrão + fibras solúveis, observou-se uma manutenção desregulada no controle da glicemia ao longo de 5 semanas de experimento, onde os níveis de glicemia se alteravam ao longo das semanas. Além disso, a dieta com fibras solúveis mostrou menor eliminação das fezes e maior ganho de peso, não contribuindo para a diminuição dos níveis de glicemia e combate à obesidade. A aveia é composta em sua composição por fibras solúveis e insolúveis, podendo servir como fonte de fibras na dieta, todavia, sugere-se estudos mais completos para analisar as mais variadas questões envolvidas nesse processo digestivo. Os efeitos fisiológicos das fibras variam, conforme estudos experimentais relatados, desde a modulação da função gastrointestinal e morfologia a alterações do metabolismo de nutrientes e aumentos das respostas imunológicas. $\mathrm{O}$ tipo de fibra e outros componentes da alimentação podem influenciar na quantidade e composição da microflora intestinal, assim como a constituição da microflora pode influenciar no perfil de ácidos graxos de cadeias curtas, produzidos pelo processo fermentativo e também influenciar na excreção de minerais e no volume das fezes por diferentes efeitos sobre trânsito intestinal.

Fietz e Salgado (1999), Santos Júnior (2003) e Trombeta e Gutkoski (1999) tembém relataram o contrário sobre as fibras solúveis, observando que no trato gastrointestinal estas retardaram o esvaziamento gástrico por possuírem facilidade 
em se incorporar à água e formar geis, tornando esse processo mais lento e conferindo maior saciedade. Com isso a ingesta de alimentos diminui o que resulta em menor ganho de peso, contrapondo em partes os achados de Chaud (2017).

A fibra tem capacidade de promover a retenção de água e aumentando o peso do bolo fecal. Com o volume aumentado, há o amolecimento das fezes na luz do intestino, promovendo peristaltismo e consequentemente o aumento da progressão fecal, da frequência de evacuações, da excreção de sais biliares, esteróides e gorduras, que têm ação catártica, ou seja, têm efeitos semelhantes aos medicamentos que aumentam a evacuação e provocam purgação. Tem sido demonstrado que muitas fontes de fibras podem reduzir o tempo de trânsito gastrointestinal, mas o efeito preciso vai depender do tipo e da forma de fibra que é ingerida. Pelo seu alto índice de fibras, os farelos de cereais, incluindo a aveia, vêm ganhando espaço na terapêutica da constipação intestinal. Por esta razão, vários produtos à base desses farelos têm sido desenvolvidos pela indústria alimentícia, destacando-se a aveia e o farelo de milho (REIS, 2003).

\subsection{AVEIA E DISLIPIDEMIAS}

As principais causas de mortes em todo mundo, segundo a Organização Mundial da saúde (2011) são as doenças cardiovasculares. Estima- se que 17,3 milhões de pessoas morreram por Doenças Cardiovasculares. A hipertensão arterial sistêmica, tabagismo, dislipidemias, obesidades, sedentarismos e diabetes mellitus (CASTRO et al, 2004). A ingestão de fibras exerce um papel preventivo contra essas doenças, em particular as fibras solúveis (DELANEY COLABORADORES, 2003).

A B-glucana, fibra solúvel presente na aveia, traz grandes benefícios para a saúde auxiliando na redução do perfil lipídico, do colesterol, na redução da lipoproteína de baixa densidade (LDL), no aumento da lipoproteína de alta densidade (HDL) e também melhora da glicemia pós -prandial (CASIRAGHI COLABORADORES, 2006; MIRA, GRAF E CÂNDIDO, 2009).

Wolever et al (2010), em um estudo multicêntrico, de dupla paralelação e desenho paralelo com um total de 345 pessoas, administraram diferentes concentrações de B-glucana em diferentes produtos, avaliando também o seu peso molecular e potencial de redução, concluindo que a B-glucana auxilia na redução de colesterol plasmático, colesterol total, LDL, e aumenta a viscosidade intestinal, também reduzindo a reabsorção de ácidos biliares, levando a menor níveis de lípides plasmáticos e maior presença de ácidos graxos nas fezes.

Wood et al (2000), através de uma análise de diferentes pesquisas sobre a avaliação do papel da concentração e do peso molecular da B-glucana da aveia na determinação do efeito da viscosidade na glicose plasmática e insulina após uma carga oral de glicose relataram que há uma correlação significativa entre os níveis de pico de concentração de glicose no sangue e a alta viscosidade de B-glucana. Tong et al (2015), também encontraram através da ingestão de outro ceral, a cevada sem casca, a redução na concentração de LDL no plasma e de colesterol em hamsters hipercolesterolêmicos em correlação à presença de B-glucana.

Ban et al (2015), também encontraram diminuições plasmáticas e hepáticas pelo uso de B-glucana nos níveis de colesterol e LDL em ratos Sprague-Dawley alimentados com uma dieta hipercolesterolemiante. O estudo também encontrou que a aveia cozida favoreceu a melhora do metabolismo do colesterol, aumentando as excreções fecais de lipídeos, colesterol e ácidos biliares. Além disso, o efeito hipocolesterolêmico da aveia cozida também se correlacionou diretamente com o seu maior teor de B-glucana. 
Chang et al (2013) realizaram um estudo com indivíduos de IMC $\geqq 27$ e idade entre 18 e 65 anos, divididos aleatoriamente em um grupo controle ( $n=18$ ) e um grupo tratado com aveia $(n=16)$, tomando um placebo ou cereal de aveia contendo B-glucana, respectivamente, por 12 semanas e mostraram que a aveia sua B-glucana auxiliaram na redução do peso corporal, na diminuição de gordura corporal e melhora na relação de circunferência de cintura-quadril em indivíduos adultos com idade entre 18 e 65 anos e Índice de Massa corporal maior que 27, sendo que estes indivíduos também mostraram melhora da função hepática, aumento dos níveis de HDL e diminuição dos níveis de LDL.

Além dos estudos citados, outros inúmeros estudos tem demonstrado que a aveia e seus compostos nutricionais (principalmente a B-glucana) tem surtido melhora no peril lipídico em humanos e em ratos, sugerindo assim que a adesão deste alimento na dieta de pessoas com dislipidemias é de grande valia, auxilando consequentemente na redução do risco de doenças cardiovasculares e suas complicações.

\section{PRESENÇA DE GLÚTEN NA AVEIA}

Glúten é definido por ser uma substancia elástica, insolúvel em água, aderente e responsável pelas estruturas das massas dos alimentos. Ele é formado pelas ligações químicas das proteínas denominadas prolaminas, principalmente a gliadina e a glutenina. A proteína resultante geralmente se encontra presente em cereais com trigo, centeio, cevada e até mesmo na aveia pela contaminação residual cruzada relacionada a produção da mesma (ARAÚJO, et al., 2010).

Em alguns indivíduos a ingestão do glúten pode desencadear uma resposta imunológica causando uma patologia chamada "doença celíaca", através de um mecanismo não imediato por imunoglobulina $\mathrm{E}(\mathrm{lgE})$, resultando em alergia (HISCHENHUBER C, et al, 2006). Quando ingerido o glúten, ocorre o contato com as células intestinais, levando a uma resposta imune e também à limitação da área disponível para absorção de nutrientes (THOMPSON et al, 2005).

A ingestão de cereais ocorre por via oral, por via inalatória, ou por via transdérmica pelo contato com produtos dermatológicos que contenham a presença do glúten em sua composição através da adesão de cerais à formula, como é o caso dos emolientes, ocorrendo a sensibilização ( BATTAIS F, et al, 2003). A maioria dos indivíduos sensibiliza-se durante o primeiro ano de vida, evoluindo para a tolerância imunológica aos três a cinco anos de idade (SAPONE A, et al, 2012).

Existem estudos que relataram pacientes com doença celíaca podendo tolerar quantidades moderadas de aveia em sua dieta (JANATUINEN et al, 1995, HOFFENBERG et al, 2000; PICARELLI et al, 2001; KILMARTIN et al, 2003).

Stine Størsrud et al (2003), avaliaram os efeitos da inclusão de grandes quantidades de aveia na dieta de pacientes celíacos quanto à qualidade de nutrientes, biodisponibilidade de $\mathrm{Fe}$, quantidades de $\mathrm{Fe}$ disponíveis, estado nutricional e ocorrência de sintomas gastrintestinais (Gl). A investigacao durou 2 anos, e contou com 20 participantes maiores de 18 anos que ingeriram uma média de 93g/dia. Eles concluíram que a inclusão de aveia na dieta livre de glúten dos pacientes celíacos teve efeitos benéficos em pacientes adultos com doença celíaca em remissão, entre os benefícios estao o efeito saciante, regularidade na função do intestino, fornecimemto de energia. É importante notar que nenhum efeito negativo foi visto nos níveis de hemoglobina e ferritina sérica após a introdução da aveia. A flatulencia dos indivíduos aumentou (devido às fibras) inicialmente durante o estudo, e diminuiu 
quando o intestino se adaptou para a maior ingestão de fibras. Foi concluído que pode haver a permissao de aveia na dieta, e que esta teve boa aceitação, sendo que 15 dos 20 pacientes permaneceram ao longo dos dois anos consumindo a aveia, e 5 permaneceram por seis meses. Esse estudo mostrou que pode-se aumentar o valor nutricional de uma dieta que deveria ser inicialmente livre de glúten, bem como fazer a dieta mais agradável para os pacientes e assim aumentar a adesão ao tratamento.

Lundin et al (2003) avaliaram 19 pacientes adultos com doença celíaca que estavam em uma dieta isenta de glúten e comecaram a ingerir $50 \mathrm{~g}$ de aveia por dia durante 12 semanas. A aveia foi bem tolerada pelos pacientes, porém alguns pacientes apresentaram dores abdominais e inchaços, e 5 dos pacientes apresentaram níveis positivos de interferon y mRNA, sendo que o IFN-y serve como um sinal de ativação de células $T$, porém pode não ser diretamente responsável pela atrofia das vilosidades vistas na doença celíaca. Ainda, um dos pacientes desenvolveu atrofia vilositária parcial e erupção cutânea durante um primeiro desafio com aveia. Subsequentemente, esse paciente melhorou com uma dieta isenta de aveia, mas desenvolveu atrofia vilositária subtotal e dermatite dramática durante um segundo desafio, mantendo o grau de intolerância. O estudo concluiu seguranca na ingestao de aveia para os celíacos avaliados, mas também que se deve ter atenção com o consumo de produtos de aveia, para garantir que estes estejam livres de contaminação cruzada com a presença de glúten. Mesmo com boa aceitação dos pacientes celíacos à aveia, esta pesquisa também nos mostrou que se deve ter uma atenção ao consumo desta na dieta desses pacientes, não excluindo totalmente possíveis complicações pelo consumo da aveia.

Outro estudo feito por Nijeboer (2006) publicado no Scandinavian Journal of Gastroenterology, avaliou crianças com doença celíaca e seus anticorpos anti-avenina (anticorpor circulantes para aveia e avenina). O estudo avaliou 116 criancas divididas em grupo padrao e o grupo consumindo pelo menos $10 \mathrm{~g}$ de aveia diariamente. Foi concluído que houve uma diminuição significativa nos anticorpos anti-avenina em ambos os grupos no final, em comparação com o início do estudo, ( $p<0,001)$, mas nenhuma diferença foi encontrada entre os dois grupos.

\section{CONSIDERAÇÕES FINAIS}

A aveia tem uma grande importância na produção agrícola do Brasil, principalmente na região Sul. Este cereal é utilizado de várias formas, desde o consumo humano até à fabricação de cosméticos e uso na alimentação animal.

O consumo de aveia tem sido associado a redução do risco e a apresentação de melhora no quadro de diversas doenças como o diabetes; complicações hiperglicêmicas; dislipidemias e hipercolesterolemias, reduzindo assim o risco de doenças cardiovasculares; distúrbios gastrointestinais; melhora de funções imunológicas e auxílio no controle de sobrepeso e obesidade.

A aveia pode possuir através da contaminação cruzada a presença das prolaminas gliadina e glutenina, que unidas formam o glúten, este que tem efeito alergênico em alguns indivíduos. Isso se dá devido ao cultivo e processamento da aveia ser realizado nos mesmos locais onde se produz outros cerais como o trigo e a cevada que possuem estes nutrientes em sua composição. Todavia, estudos tem demonstrado que os efeitos alergênicos causados por estes compostos na aveia tem apresentado menores efeitos negativos se comparada a outros cereais que também possuem o glúten em sua composição. Com isso, a aveia também se apresentou tolerável por pacientes com Doença Celíaca, trazendo qualidade nutricional para a dieta destes 
indivíduos sem que estes tenham algum tipo de reação controversa na saúde e assim podendo se beneficiar do consumo da mesma, todavia, as reações negativas ao consumo deste cereal vão depender do grau de tolerância de cada indivíduo sendo necessário mais estudos à respeito dessa questão e maior atenção ao consumo, tendo preferência pelo consumo de um alimento livre de contaminação cruzada.

A presença de B-Glucana, de antioxidantes e compostos fenólicos (principalmente os flavonoides), dá a aveia o status de alimento funcional, sendo que estas substâncias juntamente com uma composição nutricional rica em fibras, proteínas, ácidos graxos de cadeia curta e ainda um baixo índice glicêmico relacionado aos carboidratos presentes trazem à esse alimento uma posição de destaque na dieta do ser humano com diversos efeitos fisiológicos sobre a saúde dos mesmos.

A aveia pode ser empregada à alimentação humana de diversas formas, sendo assim, a mesma poderá proporcionar efeitos diferentes ao organismo humano e suas mais variadas situações fisiológicas apresentadas, dependendo de como este alimento é apresentado ou preparado. A aveia sofre mudanças em sua composição nutricional de acordo com o local de seu plantio, das condições climáticas, do tratamento do solo, do tratamento térmico para o consumo e da forma como este é apresentado em diversos produtos do cotidiano. Com isso os nutrientes que podem apresentar benefícios em determinadas funções fisiológicas poderão se apresentar em diferentes concentrações e surtir diferentes efeitos ao organismo no alimento dependendo de como o alimento será produzido, preparado e consumido.

Desta maneira, sugere-se que sejam realizadas mais pesquisas sobre o consumo da aveia e seus compostos compostos nutricionais, principalmentes sobre os compostos antioxidantes e fenólicos que carecem de mais estudos, para que se obtenha maiores informações sobre os efeitos da mesma na alimentação e na saúde humana.

\section{REFERÊNCIAS}

ANVISA - Agência Nacional de Vigilância Sanitária. Alimentos com Alegações de Propriedades Funcionais e ou de Saúde, Novos Alimentos/Ingredientes, Substâncias Bioativas e Probióticos. IX - Lista de alegações de propriedade funcional aprovadas. 2008.2 Disponível em: http://www.anvisa.gov.br/alimentos/comissoes/tecno_lista_alega.htm. Acesso em 31 de Março de 2019.

BAN, Y. et al. Effects of different cooking methods of oatmeal on preventing the diet-induced increase of cholesterol level in hypercholesterolemic rats. Lipids in health and disease, v. 14, n. 1, p. 135, 2015.

BATTAIS, F.; PINEAU, F.; POPINEAU, Y.; APARICIO, C.; KANNY, G.; GUERIN, L.; MONERET-VAUTRIN, D. A.; DENERY-PAPINI, S. Food allergy to wheat: identification of immunoglobulin $E$ and immunoglobulin G-binding proteins with sequential extracts and purified proteins from wheat flour. Clin Exp Allergy. 2003;33:962-70.

BODINHAM, C. L. et al. Peptídeo-1 semelhante ao glucagon plasmático endógeno após consumo agudo de fibra dietética. British Journal of Nutrition , v. 110, n. 8, p. 1429-1433, 2013. 
BRANDÃO, A. P.; et al. I Diretriz Brasileira de Diagnóstico e Tratamento da Síndrome Metabólica. Arquivos Brasileiros de Cardiologia. São Paulo, SP, v. 84, abr 2005.

BRASIL: Ministério da Saúde. Secretaria de Atenção à Saúde Coordenação-Geral da Política de Alimentação e Nutrição. Guia alimentar para a população brasileira. Série A. Normas e Manuais Técnicos. Brasília, DF, 2005.

BRENEMAN, C.B.; TUCKER, L.; Fibra dietética: consumo de fibra e resistência a insulina papel de gordura corporal e physactividade ical. British Journal of Nutrition. 2013; 110 (02): 375-83

BRENNAN, C.S. Dietary fibre, glycaemic response, and diabetes. Mol. Nutr. Food Res. 2005, 49, 560-570. [CrossRef] [PubMed]

CHANG, $\mathrm{H}-\mathrm{C}$, et al. Oat prevents obesity and abdominal fat distribution, and improves liver function in humans. Plant foods for human nutrition, v. 68, n. 1, p. 18-23, 2013.

CHAUD, S. G et al. Efeitos produzidos pela ingestão de fibras alimentares: solúveis e insolúveis em camundongos. Ciência ET Praxis, [S.I.], v. 2, n. 03, p. 5560, abr. 2017. ISSN 1983-912X. Disponível em: <http://revista.uemg.br/index.php/praxys/article/view/2087/1081 >. Acesso em: 02 Abr. 2019.

CHRISTENSEN, K. L.; HEDEMANN, M. S.; LAERKE, H. N.; JORGENSEN, H.; MUTT, S. J. .; HERZIG, K. H. et al.. Journal of Agricultural and Food Chemistry. 2013;

CRESTANI, M. et al . Conteúdo de $\beta$-glucana em cultivares de aveia-branca cultivadas em diferentes ambientes. Pesq. agropec. bras., Brasília, v. 45, n. 3, p. 261-268, Mar. 2010. Available from <http://www.scielo.br/scielo.php?script=sci_arttext\&pid=S0100-

204X2010000300005\&lng=en\&nrm=iso >. access on 12 Mar. 2019. http://dx.doi.org/10.1590/S0100-204X2010000300005.

SILVA, A. L. V, et al. Beta Glucana da Aveia (Avena Sativa) E Sua Relação com o Diabetes Mellitus. International Journal of Nutrology, v. 11, n. S 01, p. Trab212, 2018.

DE MORI, C.; FONTANELI, R. S.; SANTOS, R.P; "Aspectos econômicos e conjunturais da cultura da aveia." Embrapa Trigo-Documentos (INFOTECAE)(2012).

DYKES, L.; ROONEY, L. W. Compostos fenólicos em grãos de cereais e seus benefícios para a saúde. Cereal foods world , v. 52, n. 3, p. 105-111, 2007.

EVERT, A.B.; BOUCHER, J. L.; CYPRESS, M.; DUNBAR, S. A.; FRANZ, M. J.; MAYER-DAVIS, E. J.; NEUMILLER, J. J.; NWANKWO, R.; VERDI, C. L.; URBANSKI, $P$.; et al. Nutrition therapy recommendations for the management of adults with diabetes. Diabetes Care 2014, 37 (Suppl. S1), S120-S143. [CrossRef] [PubMed] 
FIETZ, V. R.; SALGADO, J. M.. Efeito da pectina e da celulose nos níveis séricos de colesterol e triglicerídeos em ratos hiperlipidêmicos. Rev. Ciência Tecnol. Aliment. v. 19. n. 3. Campinas, set./dez. 1999.

FOOD AND DRUG ADMINISTRATION (FDA), CODE OF FEDERAL REGULATIONS. Available online: http://www.ecfr. gov/cgi-bin/textidx?SID=23f8ec3719c5d0e75285aa894ab1e5b0\&mc=true\&node=se21.2.101_181\&r gn=div8 (accessed on 14 September 2015).

GUTKOSKI, L. C.; PEDÓ, I. Aveia: composição química, valor nutricional e processamento. São Paulo: Varela, 2000. 191 p.

GUTKOSKI, L.C.; EL-DASH, A.A.; PEDÓ, I. Caracterização química e nutricional de frações de moagem de aveia. Arquivos de Biologia e Tecnologia, v. 40, n. 1, p. 121-134, 1997.

HISCHENHUBER, C.; CREVEL, R.; JARRY, B.; MÄKI, M.; MONERET-VAUTRIN D. A.; ROMANO, A.; TRONCONE. R.; WARD, R.; Review article: Safe gluten limits in wheat allergy or coeliac disease. Aliment Pharmacol Ther. 2006;23(5):559-75.

HOFFENBERG E, HAAS J, DRESCHER A, et al. (2000) A trial of oats in children with newly diagnosed coeliac disease. J Pediatr 137, 361-366.

JACINTHO, T. M. Qual é a ingestão de fibras alimentares pelo brasileiro? Medicina Cursos, Dez, 2007.

JANATUINEN, E.K.; PIKKARAINEN P.H.; KEMPPAINEN T.A, et al. (1995) A comparison of diets with and without oats in adults with coeliac disease. New Eng J Med 333, 1033-1037

JENKINS, D.; WOLEVER, T.; JENKINS, A. L.; GIORDANO, C.; GIUDICI, S.; THOMPSON, L. U et al. O American Journal of Clinical Nutrition. 1986

KILMARTIN, C.; YNCH, S.; ABUZAKOUK, M.; WIESER H. \& FEIGHERY C. (2003) Avenin fails to induce a Th1 response in coeliac tissue following in vitro culture. Gut 52, 47-52.

LEONARD, M. M.; VASAGAR, B. Perspective on gluten-related diseases. Clin Exp Gastroenterol. 2014;7:25-37.

LU, Z. X.; WALKER, K. Z.; MUIR, J.G.; O'DEA, K. Arabinoxilano fibra melhora o controle metabólico em pessoas Tipo 1. Lu ZX, Walker KZ, Muir JG, O'Dea K. Arabinoxilano fibra melhora o controle metabólico em pessoas Tipo II diabetes. European Journal of Clinical Nutrition. 2004; 58 (4): 621-8.

LUDWIG, D.S. The glycemic index: physiological mechanisms relating toobesity, diabetes, and cardivascular disease. JAMA. 2002; 287 (18): 2414-23.

LUNDIN, K.; NILSEN, E. M; SCOTT, H. G, et al. Oats induced villous atrophy in coeliac disease Gut. 2003;52:1649-1652. 
MILANE, L.; JORDÃO, Í. S. C. Utilização de fibras no tratamento dietoterápico da Síndrome Metabólica. RBONE-Revista Brasileira de Obesidade, Nutrição e Emagrecimento, v. 2, n. 12, 2012.

MOLIN, V. T. S. Chemical And Sensory Evaluation Of Oats In Different Forms Of Processing. 2011. 80 f. Dissertação (Mestrado em Ciência e Tecnologia dos Alimentos) - Universidade Federal de Santa Maria, Santa Maria, 2011.

NIJEBOER, P.; BONTKES, H. J.; MULDER, C. J.; BOUMA, G.; Non-celiac Gluten Sensitivity. Is it in the Gluten or the Grain?. J Gastrointestin Liver Dis. 2013;22(4):435-40.

PACHECO, M.T.B.; SGARBIERI., V.C. Fibra e doenças gastrointestinais. In: LAJOLO, F.M. et al. Fibra Dietética en Iberoamérica: Tecnologia Y Salud. São Paulo: Editora Varela, 2001. 469p.

PEREIRA, S.C.L. et al. Desenvolvimento de um biscoito tipo cookie a base de soja/aveia e avaliação de seus efeitos metabólicos em ratos diabéticos. Revista do Médico Residente, v. 13, n. 2, 2011.

PHILIPPI, ST. Pirâmide dos Alimentos, $1^{\circ}$ ed. São Paulo; Manole, 2008.

PICARELLI, A.; DI TOLA, M.; SABBATELLA, L et al (2001). Immunologic evidence of no harmful effect of oats in celiac disease. Am J Clin Nutr 74, 137-140

REBELLO, C. J.; BURTON, J.; HEIMAN, M.; GREENWAY. FL.; Gastrointestinal microbiome modulator improves glucose tolerance in overweight and obese subjects: A randomized controlled pilot trial. Journal of diabetes and its complications. 2015; 29(8):1272-6.

REIS, N. T. Nutrição Clínica: Sistema Digestório. Rio de Janeiro: Rubio, 2003. 294p

REYES, F.G. \& AREAS, M. A. Fibras alimentares e metabolismo de carboidratos. In: LAJOLO, F.M. et al. Fibra Dietética en Iberoamérica: Tecnologia Y Salud. São Paulo: Editora Varela, 2001. 469p.

SANTOS, J.; Laxantes e Purgativos - O paciente e a constipação Intestinal. Rev. Coloproct, out. 2003. v. 23. n.2

SAPONE, A.; BAI, J. C.; CIACCI, C et al. Spectrum of gluten - related disorders: consensus on new nomenclature and classification. BMC Med. 2012;10:13.

SHAW, J.E.; SICREE, R.A.; ZIMMET, P.Z. Global estimates of the prevalence of diabetes for 2010 and 2030. Diabetes Res. Clin. Pract. 2010, 87, 4-14. [CrossRef] [PubMed]

SILVA, F.M.; MELLO, V.D. Fator de Índice Glicêmico e Carga Glicêmica no manejo do Diabetes Melitus. Rev HCPA, v.26, n.2, p.73-81, 2006. 
SOCIEDADE BRASILEIRA DE DIABETES. Consenso brasileiro sobre Diabetes 2002. Diagnóstico e classificação do diabetes melito e tratamento do diabetes melito tipo 2. Rio de Janeiro: Diagraphic; 2003.

SOUZA, A. M.; OSUGUE, J. Y. Estudo das alterações morfológicas no epitélio do intestino grosso em dois grupos de ratos alimentados com farinha de aveia e farinha refinada. Cadernos UniFOA, v. 6, n. 2 Esp, p. 83, 2018.

STØRSRUD, S., HULTHÉN, L., \& LENNER, R. (2003). Beneficial effects of oats in the gluten-free diet of adults with special reference to nutrient status, symptoms and subjective experiences. British Journal of Nutrition, 90(1), 101-107. doi:10.1079/BJN2003872

THOMPSON, T.; DENNIS, M.; HIGGINS, L. A.; LEE, A. R.; SHAVRETT, M. K. Glutenfree diet survey: are Americans with celiac disease consuming recommended amounts of fibre, iron, calcium and grain foods? J Hum Nutr Diet. 2005; 18:163-9.

TONG, L. T.; ZHONG, K.; LIU, L.; ZHOU, X.; QIU, J.; ZHOU, S. Effects of dietary hull-less barley b-glucan on the cholesterol metabolism of hypercholesterolemic hamsters. Food Chem. 2015;169:344-9.

UNICAMP, NEPA. TACO: tabela brasileira de composição de alimentos. Campinas: UNICAMP-Universidade Estadual de Campinas, 2011.

WANG, Q.; ELLIS, P.R. Oat $\beta$-glucan: Physico-chemical characteristics in relation to its blood-glucose and cholesterol-lowering properties. Br. J. Nutr. 2014, 112, S4-S13. [CrossRef] [PubMed]

WOLEVER T. M. S.; TOSH S.M.; GIBBS A. L.; BRAND-MILLER J.; DUNCAN A. M.; HART, V et al. Physicochemical properties of oat $\beta$-glucan influence its ability to reduce serum LDL cholesterol in humans: a randomized clinical trial. Am J Clin Nutr. 2010; 92:723-32.

WOOD, P. J.; BEER, M. U.; BUTLER G. Evaluation of role of concentration and molecular weight of oat $\beta$-glucan in determining effect of viscosity on plasma glucose and insulin following an oral glucose load. Brit J Nutr. 2000;84:19-23.

ZHANG, P.; ZHANG, X.; BROWN, J.; VISTISEN, D.; SICREE, R.; SHAW, J.; NICHOLS, G. Global healthcare expenditure on diabetes for 2010 and 2030. Diabetes Res. Clin. Pract. 2010, 87, 293-301. [CrossRef] [PubMed]

ZHOU, M.X.; HOMES, G.M.; ROBARDS, K.; HELLIWELL, S. Fatty acid composition of lipids of Australian oats. Journal of Cereal Science, New York, v. 28, n. 980212, p. 311-319, 1998. 